\title{
Sechs Briefe Albrecht von Hallers
}

Von Gerrit A. Lindeboom

Erich Hintzsche zum Gedächtnis

Die «Hollandsche Maatschappij der Wetenschappen» wurde im Jahre 1752 von einigen maßgebenden Gelehrten in Haarlem gegründet. Diese wissenschaftliche Gesellschaft ist die älteste der jetzt noch bestehenden in Holland. Sie war von vornherein darauf bedacht, Beziehungen mit ausländischen Gelehrten anzuknüpfen und sie zum Mitglied zu ernennen. Im Jahre 1764 wurde Albrecht von Haller zum Mitglied erwählt. Dieses Zeichen der Anerkennung seiner hohen wissenschaftlichen Verdienste muß dem gefeierten Gelehrten vielleicht auch gerade darum willkommen gewesen sein, weil es aus Holland kam.

Er hatte nämlich in seiner Jugend während zwei Jahren (1725-27) in Leiden unter Boerhaave studiert und sofort dessen Größe erkannt. Später hat er etliche Gelegenheiten benutzt, um seiner Bewunderung für den «vir incomparabilis», den "praeceptor Europae» Ausdruck zu geben. Jedoch stand er nicht in herzlichen Beziehungen zu der Leidener Fakultät nach Boerhaaves Tod im Jahre 1738. Er hat, sei es anonym, kritische Bedenken gegen Boerhaaves anatomische Kenntnisse geäußert (Lesky, 1958) und dessen Vorlesungen über die Institutiones Medicae nach dem Tode des berühmten Lehrers herausgegeben (Haller 1739-44). Diese Ausgabe hatte zwar großen Erfolg; aber sie stand deutlich in schroffem Widerspruch zu den Wünschen Boerhaaves, der zeitlebens die unautorisierte Veröffentlichung seiner Vorlesungen mehrmals scharf verurteilt hatte. Gerade darum muß Haller sich gefreut haben, Mitglied einer holländischen Gesellschaft von Gelehrten zu werden.

Die Briefe befinden sich noch in dem Archiv der Gesellschaft in Haarlem. Sie sind (mit Ausnahme von einem) geschrieben in der bekannten feinen, nach zwei Jahrhunderten aber etwas verblaßten Handschrift und haben keinen medizinischwissenschaftlichen Wert, da sie sich nur beziehen auf die Abfassung des an Haller auszustellenden Diploms und auf die Herstellung eines Beitrags von Haller zu den wissenschaftlichen Veröffentlichungen der Gesellschaft. Diese erschienen seit 1753 unter dem Titel «Verhandelingen, uitgegeeven door de Hollandsche Maatschappye der Wetenschappen, te Haarlem» (Abb.1).

Haller hat einen großen Aufsatz von fast 100 (gedruckten) Seiten über das Gehirn von Vögeln und Fischen geschickt. Das Manuskript war in lateinischer Sprache verfaßt und wurde ins Holländische übersetzt von F.W. de Monchy (1749-1796), Stadtarzt in Rotterdam, der nachher 1780 seinerseits Mitglied der 


\section{VERHANDELINGEN,}

UITGEGEEVEN DOOR DE

\section{H O L LA N D S C HE \\ MAATSCHAPPYE}

D E R

\section{WEETENSCHAPPEN, \\ T E}

H A A R L E $\mathrm{M}$,

X. DEELS I. STUK.

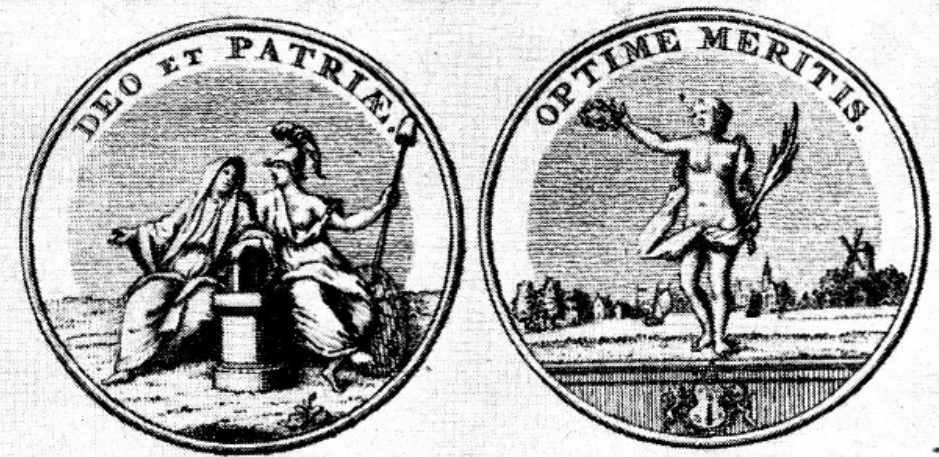

\section{TE H A A R L E M, By $\quad$. $B \quad O \quad S \quad C \quad H$. \\ Drukker van de Hollandiche Maatfehappye der Weetenfchappen, 1768.}

Mat Privilegie der El. Gr. Mog. Heeren Statin wan Hollawl en Wefterieshav.

Abb.1. Titelblatt des 10. Bandes der «Verhandelingen» der Haarlemer Gesellschaft 


\section{VERHA N DELING \\ O V E R D E}

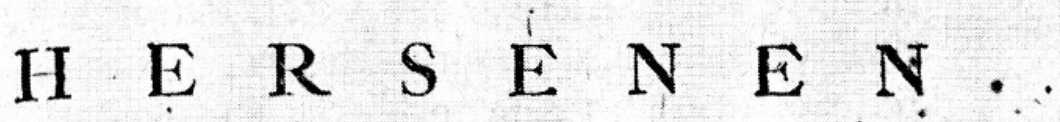

D E R

\section{- VOGELEN EN VISSCHEN.}

D $\quad 0 \quad 0 \quad$ R

\section{ALBERTUS VAN HALLER,}

- Uit het Latyo vertaald door

FRANCQIS WILLEM DE MONCHX.

\section{Toorheen heb ik reeds eene Befchry-} ving ( $a$ ) van de Herfenen der Visfchen gegeven; maar zo, dat ik dezelve van allen by één genomen had.

\section{. Dus}

(a) Eleneat. Pbyfolog. Tom. IV. pag. 591.

\section{E}

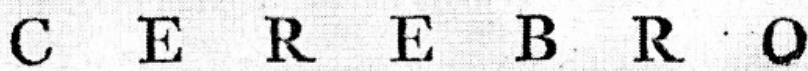

\section{AVIUM ET PISCIUM.}

P iscium equidem Cerebra olim defcripta dedi (*) ita

tamen, ut omnium hiftoriam iv unam conjungerem.

(*) Element. Pbyfiolog. Tom. IV. pag. 59r.

Cum

T 2

Abb.2. Erste Seite von Hallers Aufsatz über das Gehirn der Vögel und der Fische 
Haarlemer Gesellschaft werden sollte. Die niederländische Übersetzung wurde abgedruckt mit der ursprünglichen lateinischen Fassung in kleineren Buchstaben unten an der Seite (Abb.2).

Der erste Brief, worin Haller seinen Dank für die Ernennung als Mitglied ausspricht, ist offenbar nicht erhalten geblieben.

Vier der Briefe sind in französischer Sprache geschrieben, einer deutsch und einer lateinisch.

Erster Brief, den 19. Juli 1764

Haller gibt an, welche Titel seinem Namen auf dem Diplom beigefügt werden sollen.

Ma main tremblante forme assez mal des caracteres Monsieur. je vais tacher pourtant de rendre lisible.

Albert de Haller Membre du Conseil Souverain de la République de Berne, ancien Vice Gouverneur d'Aigle et Directeur des Salines de la Rep: Seigneur de Goumoens-le-Jux et du fief d'Eclagnens. President de la Société Royale des Sciences de Gottingue Membre - de plusieurs academies.

Vous pouvés envoyer le Diplome a $\mathbf{M}^{\mathrm{rs}}$ Reuss freres Epiciers. Maguister a Amsterdam.

Je renouvelle Monsieur les expressions de ma reconnaissance envers Vous, et je reconnaissai la Société, quand j'aurai reçu son diplome. Je tacherai meme de travailler pour elle.

Jai l'honneur d'étre très parfaitem ${ }^{t}$

Votre très humble et très obeissant

Serviteur Haller.

Roche, 19 Juillet 1764.

Anschrift :

A Monsieur

Monsieur van der Aa Ministre de L'Evangile Secretaire de la Societé des Sciences
Haarlem
en Hollande 


\section{Haller dankt für den Empfang des Diploms.}

Je n'ai reçu qu'hier Monsieur le diplome dont l'illustre Academie m'a honoré avec le recueil de ses lois. Je vous prie de Lui en marquer ma parfaite reconnaissance, quoique je craigne d'en etre un membre inutile, n'entendant pas assés bien la langue hollandaise pour m'exprimer. Jai l'honneur d'etre trez parfaitem $^{\mathrm{t}}$

Monsieur

Votre trez humble et trez obeissant Serviteur

Haller. Chancelier designé de l'Univ. de

Gottingue.

Berne, 21. Nov 1764.

Anschrift :

A Monsieur

Monsieur van der Aa Secretaire

de l'Academie des Sciences en

Hollande

franco Ff sur le Main

Dritter Brief, den 2. Juni 1765

Haller äußert sein Vorhaben, zu den Veröffentlichungen der Gesellschaft einen Artikel beizutragen. Er habe verschiedene junge Leute inokuliert.

J'ai reçu Monsieur l'honneur de la Votre de 23 de Mai, avec les nouvelles sur les prix de la Société. Je reçois avec beaucoup de reconnaissance la permission d'ofrir pour Vos mémoires quelques unes de mes productions. Je ne saurois pas faire la dessus des promesses bien importantes, mes places dans la magistrature me privent presque entierement de mon loisir. Comme cependant j'ai envoyé a la Société de Gottingue un mémoire assés important sur les Salines, et comme ce mémoire ne sera pas imprimé, je ne sais pas s'il pouroit vous etre de quelque usage, et en ce cas la je vous en enverrais une exacte copie. Si vos reglemens demandent absolument que les pièces, qu'on 
Vous adresse, Vous soient uniquement destinées, je tacherai alors de trouver de loisir pour Vous envoyer mes remarques sur differens yeux de poissons; ou sur le cerveau des oiseaux. Jai fait inoculer avec le succés ordinaire trois freres gentils hommes, il faut avouer qu'ils n'ont pas été malades un moment, non plus que differens autres jeunes gros des premiers maisons, que j'avois fait inoculer prudemment. On ne sait pas aperçu le moins demanche (?) que la petite verole naturelle se soit repandue après ces inoculations.

Jai l'honneur d'etre tres parf ${ }^{\mathrm{t}}$

Monsieur

Votre tres humble et tres obeissant

serviteur Haller Sgr. de Goumoens le Jux et d'Eclagnens

ancien Directeur des Salines de Roche.

Berne 2 Juin 1765

Anschrift :

A Monsieur

Monsieur Van der Aa Ministre

de l'Evangile, Secretaire de la

Societé Batavique

a Haarlem

fr. Koblence.

Vierter Brief, den 26. Februar 1766

Haller schickt den ersten Teil eines Aufsatzes für die «Handelingen» ein.

Ich ubersende Euer Hochehrwürden den ersten Theil eines aufsazes Zu dero Handlungen. Der zweyte ist beym abschreiber. Ich habe geträchtet ihn zu franquiren. Solte es nicht völlig geschehen sein, so bitte es zu melden ich werde das franco nach bezahlen lassen. Beyliegendes kurzes Schreiben bitte an seine behörde laufen zu lassen und [.. ?..] dero

ergebenst gehorsamster diener

v Haller

Bern d. 26 febr. 1766. 
Fünfter Brief, den 25. November 1766

Haller legt diesem lateinischen Brief den zweiten Teil seines Aufsatzes bei.

Ecce vir reverendissime, partem II. historiae cerebri. Diu dilata est, quod vix inveniam, qui latinis literis meas exprimat: etiam nunc neque emendatum notarium inveni, neque nitidum. Quale ad opusculum aequi bonique consulas rogo.

Si posset ad me remitti postquam belgice fuerit redditum, curarent fratres Reuss, aromatarij Amstelodamenses.

Tu vero vale, et porro fave. Bernae d. 25. Nov. 1766

Haller

\section{Übersetzung:}

Hier sehen Sie, hochachtbarer Mann, den zweiten Teil der Abhandlung über das Gehirn. Es ist eine lange Verzögerung eingetreten, weil ich kaum jemand finden konnte, der meine Schrift in lateinische Buchstaben übertragen konnte. Auch habe ich weder einen fehlerlos arbeitenden Schnellschreiber noch einen ordentlichen gefunden.

Ich bitte Sie, daß Sie über eine solche kleine Arbeit billig und richtig urteilen wollen. Wenn sie zu mir zurückgeschickt werden könnte, nachdem sie ins Holländische übersetzt worden ist, könnten die Gebrüder Reuss, Drogenhändler in Amsterdam, die Sorge dafür übernehmen.

Es ergehe Ihnen wohl, und bleiben Sie mir ergeben.

Bern, 25. November 1766.

Sechster Brief, den 16. Juni 1768 (in einer anderen Schrift)

Haller ist krank, und der Brief ist von seinem Sohn geschrieben worden. Er dankt für die Zusendung eines Buches (wahrscheinlich handelt es sich hier um den X.Band der Haarlemer «Verhandelingen», der seinen Aufsatz enthält).

Monsieur

Je saisis avec Empressement les premiers momens qu'une Longue \& mauvaise fievre me laissent libres pour Voux temoigner ma sincere reconnaissance du. Livre que voux m'avés envoyé pour mon cher Pere, a qui y'ay fait tenir sans retard la Lettre que vous luy avez fait L'honneur de Luy 
ecrire. Dés que je pourrais sortir je chercherais une occasion pour Vous envoyer le second Exemplaire du Vol IX, j'ose vous demander la meme grace pour le Vol X desquil aura paru, agréés en d'avançe mes remercimens, de meme que les Sentimens de la respectueux Estime avec laquelle

J'ay Lhonneur d'Etre

Monsieur

Votre tres humble \& tres obeissant

serviteur

Haller

[.. ?..] le 16 Juin 1768

\section{Literatur}

Bierens de Haan, J.A. (1970), De Hollandsche Maatschappij der Wetenschappen 1752-1952. Tjeenk Willink, Haarlem (mit Supplement 1952-1970).

Haller, Albrecht von (1739-1744), Hermanni Boerhaave. Praelectiones academicae, in proprias Institutiones rei medicae edidit et notas addidit--. Vanden Hoeck, Göttingen, 7 Bde.

Hintzsche, Erich (1971), Albrecht Hallers Tagebücher seiner Reisen nach Deutschland, Holland und England 1723-1727. Neue Ausgabe. Hans Huber, Bern/ Stuttgart/Wien (Berner Beiträge zur Geschichte der Medizin und der Naturwissenschaften, Neue Folge Bd. 4).

Lesky, Erna (1958), Albrecht von Haller, Gerard van Swieten und Boerhaavens Erbe. Gesnerus 15, 120-140.

Lindeboom, G. A. (1958), Haller in Holland. Het Dagboek van Albrecht von Haller van zijn verblijf in Holland (1725-1727). Koninklijke Nederlandsche Gist- en Spiritusfabriek N. V.Delft (Privatdruck).

\section{Summary}

In the year 1764 Albrecht von Haller was elected member of the "Hollandsche Maatschappij der Wetenschappen», a learned society at Haarlem. Haller wrote a long contribution on the eye of fishes for its periodical, which had to be translated from Latin into Dutch. Six letters from Haller to the secretary of the Society, concerning his appointment and his contribution, are presented.

Prof. Dr. G. A. Lindeboom

Frans van Mierisstraat 41

Amsterdam-Z.

Niederlande 